\title{
L'emploi de ressortissants étrangers en République de Serbie
}

Ljubinka Kovačević

\section{(2) OpenEdition}

1 Journals

\section{Édition électronique}

URL : https://journals.openedition.org/rdctss/1268

DOI : $10.4000 /$ rdctss. 1268

ISSN : 2262-9815

Éditeur

Centre de droit comparé du travail et de la sécurité sociale

\section{Édition imprimée}

Date de publication : 1 avril 2020

Pagination : 206-211

ISSN : 2117-4350

\section{Référence électronique}

Ljubinka Kovačević, "L'emploi de ressortissants étrangers en République de Serbie ", Revue de droit comparé du travail et de la sécurité sociale [En ligne], 1| 2020, mis en ligne le 01 novembre 2021, consulté le 11 novembre 2021. URL : http://journals.openedition.org/rdctss/1268 ; DOI : https:// doi.org/10.4000/rdctss. 1268

\section{(c) (i) (9)}

Revue de droit comparé du travail et de la sécurité sociale est mise à disposition selon les termes de la Licence Creative Commons Attribution - Pas d'Utilisation Commerciale - Pas de Modification 4.0 International. 


\section{LJUBINKA KOVAČEVIĆ}

Université de BeLgRADE

\section{L'EMPLOI DE RESSORTISSANTS ÉTRANGERS EN RÉPUBLIQUE DE SERBIE}

Les règlementations nationales sur l'emploi des étrangers reflètent (et prennent en compte) les circonstances et les besoins exprimés en Serbie en termes de pénurie ou d'excédent de travailleurs sur le marché du travail, que ce soit en général ou dans des professions spécifiques, les objectifs des politiques migratoires, démographiques, économiques et celle de l'emploi, etc. Ainsi, les premières formes d'emploi organisé d'étrangers en Serbie remontent aux années 1920, lorsque des réfugiés russes sont arrivés en Serbie après le déclenchement de la Révolution d'octobre. La situation de ces immigrants, comme celle des autres ressortissants, était régie par le Règlement sur l'emploi des travailleurs étrangers du 24 novembre 1925. Cependant, c'est le Règlement sur l'emploi de ressortissants étrangers de $1935^{1}$ qui a régi cette question de manière plus complète, comprenant entre autres des dispositions sur la délivrance du permis de travail aux étrangers, et des dispositions sur la protection du marché du travail national contre l'afflux croissant de travailleurs étrangers pendant la crise économique.

Après la Seconde Guerre mondiale, l'emploi d'étrangers fut placé sous la responsabilité de l'Administration fédérale du travail créée au sein du ministère du Travail de la République populaire fédérale de Yougoslavie, c'est-à-dire sous la responsabilité de la Direction du travail de la République populaire de Serbie. Les unités au sein de ces directions acceptaient des experts étrangers, leur délivraient l'autorisation d'effectuer certaines missions et certifiaient l'authenticité de leurs contrats de travail. Les procédures relatives à l'authentification des contrats et à la délivrance des autorisations de travail se sont poursuivies, dans la pratique, durant les décennies suivantes, mais ces tâches relevaient de la responsabilité des services de l'emploi au niveau des Républiques, alors qu'il existait par exemple des règles spéciales pour l'emploi d'étrangers dans le domaine de la construction ${ }^{2}$. Le développement de l'économie yougoslave a pour la première fois entraîné l'adoption d'une loi particulière régissant l'emploi d'étrangers : la Loi sur les conditions de création d'une relation de travail avec les ressortissants étrangers $(1978)^{3}$, qui resta en vigueur pendant 27 ans. Cette loi a introduit le régime d'autorisation d'emploi d'étrangers, de sorte que le permis de travail est devenu une condition préalable à leur emploi. En revanche, la loi n'avait pas prévu le droit de priorité à l'emploi des travailleurs serbes sur les étrangers.

2 Loi sur l'attribution de la concession pour la construction d'ouvrages de génie civil et de bâtiments à un entrepreneur étranger, Journal Officiel de la République fédérative socialiste de Yougoslavie (ci-après JO RFSY) n²4/76.

3 JO de la RSFY n¹1/78 et 64/89; Journal officiel de la République Fédérale de Yougoslavie n42/92, 24/94 et 28/96; Journal Officiel de la République de Serbie (ci-après JO RS) n¹01/2005. 
Au début du XXle siècle est apparue la nécessité de répondre aux changements survenus dans le monde du travail, consécutifs notamment à l'intensification du processus de mondialisation. Durant cette période, le Service National de l'Emploi (SNE) a délivré en moyenne environ 2500 autorisations annuelles pour l'embauche de travailleurs étrangers. Ceci démontre que la République de Serbie ne pouvait être considérée comme un pays d'immigration, d'autant que ce chiffre était bien inférieur au nombre de permis de travail délivrés aux étrangers dans les pays voisins. Cependant, partant de l'idée que cette tendance pouvait s'inverser, le législateur serbe a considéré qu'il était nécessaire de réglementer l'emploi des migrants économiques, au regard notamment des processus d'adhésion de la République de Serbie à I'Union Européenne et à l'Organisation mondiale du commerce qui présupposaient l'introduction de nouvelles solutions harmonisant le droit serbe aux normes de ces deux organisations.

C'est en ce sens que la Loi sur l'emploi des étrangers ${ }^{4}$ fut adoptée en 2014. Toujours en vigueur, ses dispositions sont conformes aux Conventions n97 et 143 de l'OIT, ratifiées par la Serbie. En outre, conformément à l'accord de stabilisation et d'association entre les Communautés européennes et leurs États membres et la République de Serbie ${ }^{5}$, la Loi sur l'emploi des étrangers respecte également la lettre des Directives 2003/109/CE et 2004/38/CE. Par ailleurs, cette loi réglemente l'emploi des étrangers ayant demandé l'asile, des personnes reconnues refugiées, ainsi que des étrangers reconnus bénéficiaires de la protection subsidiaire, sous l'influence du droit de I'UE et plus spécifiquement des Directives 2001/55/CE, 2004/81/CE, 2009/52/UE, 2011/95/UE et 2013/33/UE. Enfin, la Loi sur l'emploi des étrangers a incorporé les conditions nécessaires à l'adhésion de la République de Serbie à l'Organisation mondiale du commerce. Cela comprend l'introduction des règles sur la prestation de services par le biais de personnes physiques, qu'il s'agisse d'étrangers en situation de mobilité professionnelle au sein d'une société, de personnes demeurant temporairement en République de Serbie dans le but d'établir des contacts d'affaires ou encore d'employeurs étrangers se préparant à débuter leur activité. II en va de même pour les personnes envoyées en République de Serbie par l'employeur en vue d'y exécuter un travail à titre temporaire, ainsi que pour les professionnels indépendants - à savoir pour les travailleurs indépendants établis à l'étranger - qui, sur la base d'un contrat conclu avec l'employeur ou l'utilisateur final de services en Serbie, exercent leurs activités commerciales en Serbie.

Un amendement à la Loi sur l'emploi des étrangers ${ }^{6}$ a été adopté en avril 2019 et est entré en vigueur le $1^{\mathrm{er}}$ janvier 2020. II vise principalement à simplifier la procédure de délivrance des permis de travail aux étrangers, dans le but de développer un environnement des affaires plus favorable en Serbie. Attirer les investissements étrangers est en effet l'une des priorités du Gouvernement de la République de Serbie.

En Serbie, un double régime de permis de travail s'applique, ce qui signifie qu'un ressortissant étranger ne peut débuter une relation de travail que s'il remplit deux conditions cumulatives: la première condition porte sur la possession d'une carte de séjour temporaire ou de résidence permanente en République de Serbie, tandis que la deuxième

$4 J O R S n^{\circ} 128 / 14,113 / 17,50 / 18$ et 31/19.

$5 \mathrm{JORS} \mathrm{n}^{\circ} 83 / 08$.

6 JORS n ${ }^{\circ} 31 / 19$. 
porte sur la possession d'un permis de travail7. La carte de séjour temporaire (quelle que soit la durée du séjour) ou de résidence permanente est délivrée par le ministère de l'Intérieur, conformément aux dispositions de la Loi sur les ressortissants étrangers ${ }^{8}$, et permet de vérifier l'existence éventuelle d'obstacles à demeure en Serbie représentant une menace à l'ordre, à la sécurité ou à la santé publique.

En outre, cette condition pour l'emploi des étrangers vise également à vérifier les ressources financières avec lesquelles le travailleur subviendra à ses propres besoins et à ceux de sa famille pendant son séjour en Serbie. En revanche, le « permis de travail » est un document permettant à un étranger d'être employé en République de Serbie, délivré par le SNE à la demande de l'employeur, contrairement au « permis de travail personnel » qui est établi à la demande de l'étranger.

Le « permis de travail personnel » permet à un ressortissant étranger d'avoir un libre accès au marché du travail, d'être travailleur indépendant et d'exercer ses droits en cas de chômage. Il ne peut être délivré que dans l'un des trois cas suivants: a) si le ressortissant étranger a une autorisation de séjour permanent (le permis de travail est alors délivré pour une durée identique à celle de la carte d'identité de l'étranger); b) si le ressortissant étranger a le statut de réfugié (le permis de travail est délivré pour une durée identique à celle de la carte d'identité du demandeur d'asile); c) si l'étranger appartient à une catégorie spéciale d'étrangers. Ce dernier cas concerne plus précisément les demandeurs d'asile?, les personnes bénéficiant d'une protection temporaire, les victimes de la traite et les personnes bénéficiant d'une protection subsidiaire. En outre, le permis de travail personnel peut également être délivré à des fins de regroupement familial, à la demande d'un membre de la famille directe d'un étranger titulaire d'un visa de séjour permanent, ou à la demande d'un membre de la famille directe d'un étranger ayant le statut de réfugié et titulaire d'un visa de séjour permanent ou temporaire. II en va de même pour les étrangers membres de la famille directe d'un citoyen serbe, et pour les étrangers d'origine serbe au troisième degré de parenté en ligne directe. Dans chacun de ces cas, le permis de travail est délivré à un membre de la famille directe de l'étranger pour la durée de validité de la carte de séjour.

Par ailleurs, un étranger titulaire d'un permis de travail en République de Serbie ne peut exercer que les emplois pour lesquels il détient l'autorisation. Contrairement à un « permis de travail personnel », le " permis de travail » est délivré à la demande de l'employeur pour un emploi (ou pour un travail indépendant, ou encore pour une catégorie spéciale d'emploi) déterminé. Les catégories particulières d'emploi comprennent l'emploi d'étrangers détachés par leur employeur pour travailler temporairement en Serbie,

7 Outre les restrictions à l'emploi de travailleurs étrangers, la protection des intérêts nationaux est également assurée par l'interdiction de l'emploi de ces personnes dans l'administration publique. Voir Loi sur les fonctionnaires (JO RS n79/05, 81/05, 83/05, 64/07, 67/07, 116/08, 104/09, 99/14, 94/17 et 95/18), art. $45, \S 1$.

8 JORS n²4/18 et $31 / 19$.

9 Par exemple en 2017, 5702 personnes ont exprimé leur intention de demander l'asile en Serbie, mais seulement 280 demandes d'asile ont été soumises aux autorités compétentes. En effet, la plupart des personnes ayant exprimé leur intention de demander l'asile n'ont pas déposé de demande appropriée car elles ont quitté la Serbie entre-temps, tandis que certaines ont été hébergées dans des centres de transit en Serbie, sans documents et sans procédures légales appropriées. 
la mutation d'un étranger au sein d'une société enregistrée à l'étranger et l'emploi de professionnels indépendants.

Le permis de travail peut être délivré à un étranger titulaire d'un permis de séjour temporaire et remplissant toutes les conditions nécessaires à l'établissement d'un contrat de travail, telles que déterminées par l'employeur dans la règlementation sur l'organisation et la systématisation des emplois. Ce permis est délivré à un étranger pour la durée prévue de l'emploi ou, tout au plus, pour la durée du visa de séjour temporaire ${ }^{10}$.

Il convient néanmoins de garder à l'esprit que le droit serbe a apporté de nouvelles restrictions à ce régime de double autorisation. La première de ces limitations est l'exigence selon laquelle il n'a pas été possible à l'employeur de trouver, parmi les travailleurs nationaux, une personne possédant les qualifications nécessaires pour l'emploi, dix jours avant la présentation de la demande de permis, voire même dans un délai encore plus court dans des cas particuliers. Il en va de même si, au cours de la période susmentionnée, l'employeur n'a pas pu trouver de candidats à l'emploi parmi les personnes ayant un libre accès au marché du travail ou les étrangers titulaires d'un permis de travail personnel inscrits auprès du SNE. Le législateur est même allé plus loin en conditionnant la délivrance d'un permis de travail au fait que l'employeur, avant de soumettre la demande de permis de travail, n'ait pas licencié de travailleurs en raison de changements technologiques, économiques ou organisationnels, dans les emplois pour lesquels des permis de travail ont été délivrés.

Un étranger travaillant en République de Serbie, conformément à la Loi sur l'emploi des étrangers, dispose des mêmes droits et obligations qu'un citoyen du pays. Toutefois, le Gouvernement peut décider de limiter le nombre de ressortissants étrangers susceptibles de bénéficier d'un permis de travail, notamment en cas de perturbation du marché du travail, conformément à la politique migratoire, à la situation du marché du travail et à sa dynamique. Le quota est déterminé sur proposition du ministère chargé de l'emploi, avec l'avis préalablement obtenu du Conseil social et économique de la République de Serbie et du SNE. Cependant, le quota ne s'applique pas à l'étranger - ni à l'employeur embauchant l'étranger - qui demande un " permis de travail personnel ", sauf si le permis de travail personnel est délivré en faveur d'une catégorie spéciale d'étrangers. En outre, le quota ne s'applique pas au permis de travail sollicité en cas de mutation d'un étranger au sein d'une société.

La possibilité de délivrer un permis de travail à un étranger qui possède un visa de long séjour pour emploi contribue également à l'amélioration de la procédure de délivrance de permis de travail. Prévue par l'amendement susmentionné à la Loi sur les étrangers de 2019,

10 En 2018, le SNE a délivré un total de 8990 permis de travail à des ressortissants étrangers en Serbie comprenant 1254 permis de travail personnels et 7736 permis de travail, dont 300 permis délivrés à des étrangers ayant une résidence permanente, 7 permis délivrés à des réfugiés, 66 permis délivrés à des étrangers appartenant à une catégorie spéciale d'étrangers, 17 permis délivrés à des étrangers d'origine serbe, 856 permis délivrés à des étrangers ayant statut de membre de la famille directe d'un citoyen serbe et 8 permis délivrés à des membres de la famille directe de l'étranger. En 2018, les travailleurs étrangers titulaires d'un permis de séjour temporaire en Serbie provenaient principalement de Chine (27\%), Russie (8,6\%), Turquie (6,8\%), Ukraine $(6,5 \%)$, Roumanie (4,6\%), Italie (4,1\%), Macédoine du Nord $(3,9 \%)$, Bosnie-Herzégovine $(3,1 \%)$, Grèce $(2,8 \%)$ et Croatie $(2,5) \%)$. Voir sur ces sujets Report on the performance of the National Employment Service for 2018; Migration Governance Profile: Republic of Serbia, mai 2018, p. 20. 
cette solution vise à simplifier les procédures d'obtention d'un visa de séjour temporaire et d'un visa de long séjour basé sur l'emploi. En effet, l'employeur peut engager la procédure de délivrance d'un permis de travail devant le SNE ${ }^{11}$ lors de la procédure d'octroi d'un visa de long séjour pour emploi, cette dernière se déroulant devant la mission diplomatique ou consulaire compétente. Le permis de travail délivré sur la base d'un visa de long séjour basé sur l'emploi est octroyé, au maximum, pour la même durée que celle du visa.

L'amendement à la Loi sur l'emploi des étrangers prescrit une procédure de prolongation unifiée auprès du SNE, qu'il s'agisse du permis de travail pour un étranger en situation de mutation professionnelle au sein d'une société, ou pour un salarié détaché travaillant en Serbie. Dans les deux cas, le permis de travail peut être prolongé pour une durée maximale de deux ans, avec l'accord du ministère chargé de l'emploi et selon l'avis préalablement obtenu du ministère chargé du domaine d'activité de rattachement de l'employeur. Le SNE obtient d'office l'approbation et l'avis des ministères compétents. En consolidant la procédure d'obtention des agréments et des avis, le processus de prolongation desdits permis de travail a été considérablement facilité et accéléré, alors que, jusqu'en 2019, l'avis du ministère compétent devait être obtenu par le demandeur de la prolongation du permis de travail, avant même que soit engagée auprès du SNE la procédure d'extension du permis.

Enfin, il convient de garder à l'esprit que, dans l'esprit des dispositions de la Directive 2004/38/CE, les citoyens de I'UE bénéficieront du libre accès au marché serbe du travail à compter de la date de son adhésion, à moins que la République de Serbie ne soit dans l'obligation de respecter un traité international qui en dispose autrement. Il en va de même pour les membres de la famille de citoyens de I'UE, qui sont des ressortissants de pays tiers mais qui détiennent un visa de séjour temporaire ou un visa de séjour permanent dans ces pays, ce qui prouve leur droit à un libre accès au marché du travail. Ces personnes n'auront pas besoin d'un permis de travail pour obtenir un emploi en République de Serbie, mais devront disposer de ressources suffisantes pour subvenir à leurs besoins et à ceux de leur famille. Les citoyens de I'UE, comme les membres de leur famille non membres de I'UE, auront librement accès au marché du travail en Serbie, même s'ils perdent leur emploi (sans faute de leur part), à condition cependant que leur emploi en Serbie ait duré au moins un an, qu'ils soient inscrits auprès du SNE et qu'ils participent à des programmes de formation continue.

En revanche, conformément aux dispositions de la Directive 96/71/CE, l'employeur établi dans un État membre de l'UE, un État membre de l'Espace économique européen ou dans la Confédération suisse, pourra détacher un étranger (qui n'est pas citoyen de I'UE) pour travailler en Serbie sans permis de travail. Cette possibilité est subordonnée à la condition qu'un contrat soit conclu, d'une part avec l'employeur (ou l'utilisateur final des services), et d'autre part avec le ressortissant étranger envoyé en Serbie. En outre, des dispositions devront réglementer précisément les droits et obligations du salarié pendant son séjour en Serbie, ainsi que les questions relatives à son logement ou à sa pension. Par

11 Cela est important du fait que le SNE sera informé, par voie d'un échange direct de données officielles avec les représentants diplomatiques ou consulaires, que les conditions de délivrance d'un permis de travail à un étranger sont remplies. Cela accélèrera considérablement la procédure de délivrance des permis de travail et permettra aux étrangers de commencer à travailler dès leur arrivée en Serbie. 


\section{RéPublique de SERbie}

ailleurs, l'étranger doit disposer d'un visa de séjour et d'un permis de travail dans le pays où se trouve le siège de l'employeur étranger.

Enfin, les conditions particulières d'emploi des étrangers ne s'appliquent pas à l'étranger qui, pendant son séjour en République de Serbie, exerce un emploi saisonnier, conformément à la Loi sur l'emploi saisonnier simplifié dans certaines activités économiques ${ }^{12}$.

12 JO RS n50/18. 